\title{
Isolation of Chlamydia psittaci from a patient with interstitial keratitis and uveitis associated with otological and cardiovascular lesions
}

\author{
S. DAROUGAR, A. C. JOHN,* M. VISWALINGAM, L. CORNELL, AND \\ B. R. JONES \\ From the Virus Laboratory, Department of Clinical Ophthalmology, Institute of Ophthalmology, University \\ of London, the External Eye Diseases Clinic, Moorfields' Eye Hospital, City Road, London, and \\ the *Royal National Ear, Nose and Throat Hospital, Gray's Inn Road, London
}

SUMMARY A case history of a 15-year-old schoolgirl with fluctuating bilateral uveitis, bilateral stromal keratitis with vascularisation, and bilateral deafness associated with tinnitus and balance disturbance is described. Three years from the onset of her clinical signs she died of a sudden cardiac arrest caused by endocarditis associated with valvular and arterial lesions. Chlamydia psittaci was isolated from her conjunctiva. In her blood type-specific antichlamydial antibody at a level of $1 / 64$ against her own isolate was detected. The clinical findings in this patient were suggestive of a Cogan's syndrome. It is highly probable that the chlamydia isolated from the eyes was responsible for her various lesions.

The genus Chlamydia comprises the 2 species, Chlamydia trachomatis and C. psittaci. C. trachomatis is responsible for a variety of ocular and genital infections in man (Jones, 1961; Jones, 1964; Lancet, 1973; Lancet, 1977). C. psittaci, which is generally associated with animals, may occasionally cause ocular infections in man (Ostler et al., 1969; Schachter et al., 1968).

In recent years several cases of endocarditis associated with $C$. psittaci have been reported (Levison et al., 1971; Ward et al., 1975). The results of these reports indicate that $C$. psittaci potentially though infrequently is a pathogen for the cardiovascular system and may cause myocarditis as well as valvular lesions (Eisentein and Taubenhass, 1958; Gelfand et al., 1972).

In this report we describe the case of a patient with a longstanding interstitial keratitis and uveitis associated with marked otological symptoms and fatal cardiovascular lesions, from whom a $C$. psittaci agent was isolated.

\section{Case report}

A schoolgirl born on 11 August 1957 of GreekCypriot extraction was first seen at Moorfields Eye

Address for reprints: Dr S. Darougar, Department of Clinical Ophthalmology, Institute of Ophthalmology, Judd Street, London WC1H 9QS
Hospital on 23 August 1972, complaining of intermittent attacks of red, painful, watering eyes during the previous 2 months. Each eye had a visual acuity of $6 / 6$. In the right eye there was hyperaemic bulbar conjunctiva and anterior uveitis with a mild degree of flare and cells in the anterior chamber, but no abnormality in the fundus. The left eye appeared normal. She was treated with topical dexamethasone eye drops with temporary benefit.

The uveitis pursued a fluctuating course involving either eye at first and soon both eyes, though she was under treatment with a variety of topical steroid eye drops including dexamethasone, prednisolone, and Betnesol. Towards the end of 1973 she developed a persistent bilateral keratitis.

The keratitis was at first mild and peripheral, but soon became central and fluctuated between a diffuse central stromal haze and coarse granular midstromal lesions (Figs. 1 and 2) and a disciform keratitis with vascularisation (Fig. 3). Later she developed some granular lipid deposition (Fig. 3).

In November 1974 she was admitted to the Royal Northern Hospital, London, for further investigations. After extensive laboratory tests (see laboratory investigations) both sarcoidosis and collagen vascular disease were excluded, and she was then discharged from the hospital on treatment with indomethacin $25 \mathrm{mg} 3$ times a day.

On 28 December 1974 the patient was referred to the Royal Northern Ear, Nose and Throat Hospital 


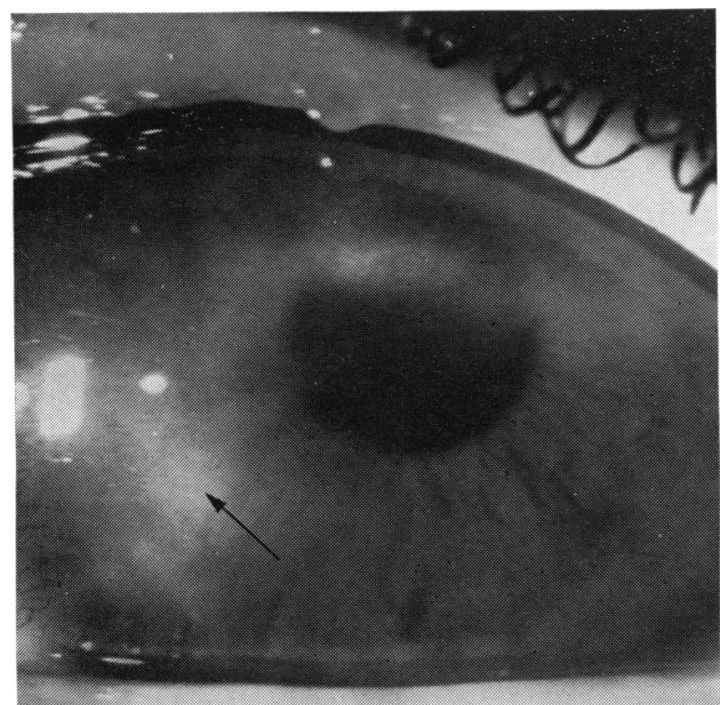

Fig. 1 Diffuse stromal keratitis

because of sudden onset of bilateral deafness, tinnitus, and balance disturbance. The deafness was of moderate severity and appeared to be symmetrical. The tinnitus took the form of a bilateral constant high pitched buzzing, and the balance disturbance, which had initially been an acute rotational vertigo, had become a constant feeling of unsteadiness, especially on sudden head movement, and it was not associated with nausea or loss of consciousness.

Clinical examination showed normal tympanic membranes, bilateral Rinne test positive, Weber test central, normal cranial nerve examination and no spontaneous nystagmus or papilloedema. There was obvious unsteadiness of gait. Pure tone audiometry showed bilateral sensorineural deafness (see Fig. 4). There was bilateral poor speech discrimination. The Bekesy audiogram was type 1 . No tone decay could be demonstrated. The short increment sensitivity test (SISI) showed 60\% response at $1000 \mathrm{~Hz}$ and $80 \%$ at $2000 \mathrm{~Hz}$. Mastoid $x$-rays and $x$-rays of the internal auditory meatuses were normal. The positional test was negative. Caloric stimulation showed a symmetrical and equal response with no evidence of canal paresis or marked directional preponderance. Electronystagmography showed no evidence of spontaneous nystagmus or any other abnormality. Electrocochleography confirmed the hearing thresholds found on pure tone audiometry. The eighth nerve action potential was of normal form.

The patient was admitted to hospital on 8 January 1975 and started on systemic prednisolone $25 \mathrm{mg}$ 3 times a day, tailing off to a maintenance dose of

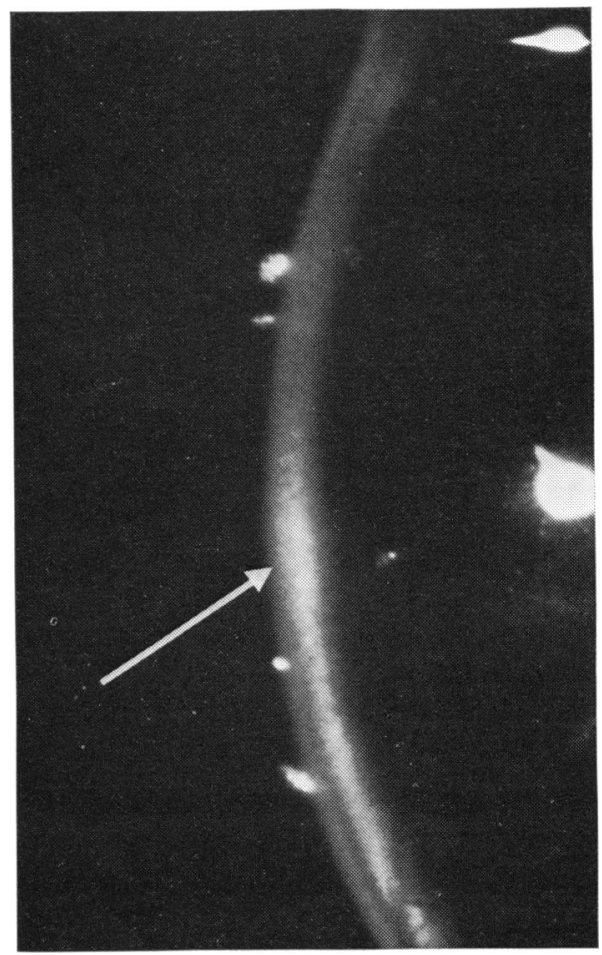

Fig. 2 Diffuse stromal keratitis in the narrow slit-lamp beam $(\times 10)$

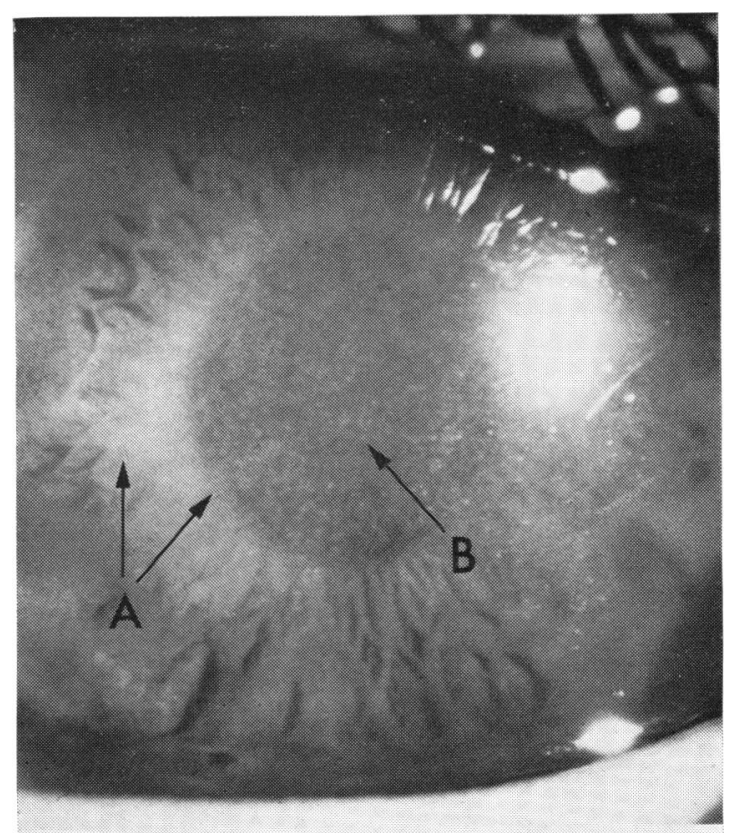

Fig. 3 Diffuse stromal keratitis (A) and granular lipid deposition $(\mathrm{B})(\times 7)$ 
Fig. 4 Pure-tone audiogram obtained on 30 December 1974. showing a moderate symmetrical sensorineural hearing loss throughout the frequency range tested

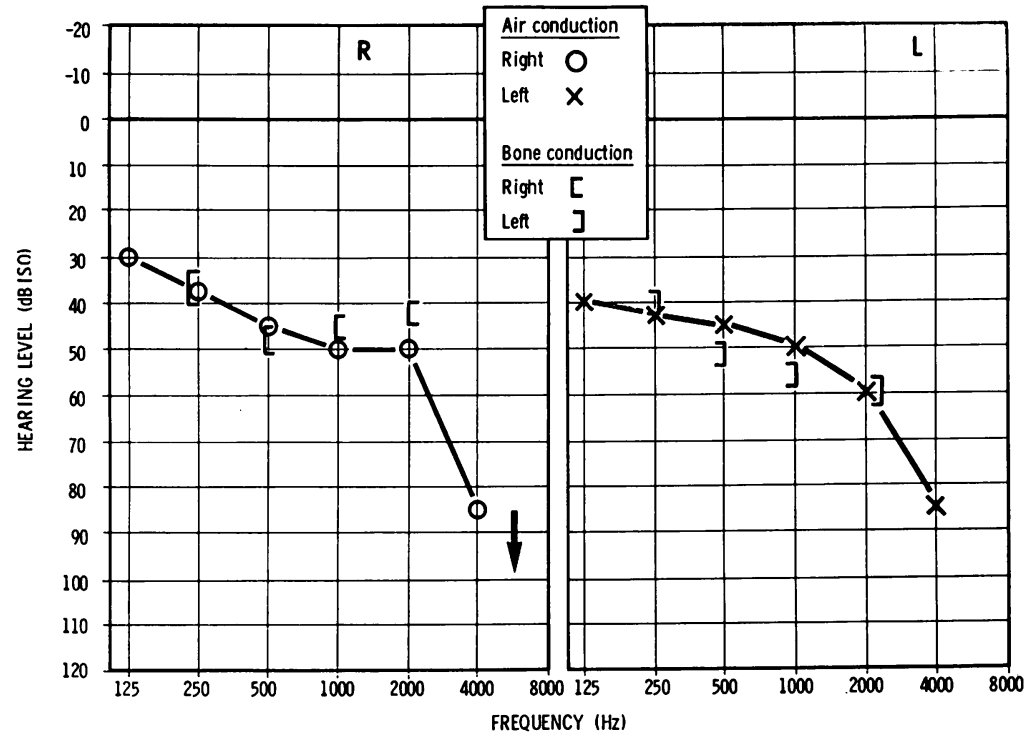

$2.5 \mathrm{mg} 3$ times a day over 2 weeks. This failed to ameliorate the otological symptoms. Over the next 10 months the deafness and tinnitus pursued a fluctuating course, but always relapsing to near their severity on presentation. The constant feeling of unsteadiness persisted with occasional exacerbations during the period of observation. However, several months later it was revealed by her father that she might have exceeded the recommended dose of indomethacin on a single occasion, though it was not clear by how much.

In February 1975 a $C$. psittaci agent was isolated from a conjunctival swabbing taken during one of the exacerbations of keratouveitis because of the resemblance to Reiter's disease. After this the patient was treated with a course of doxycycline $300 \mathrm{mg}$ as an initial dose followed by $100 \mathrm{mg}$ daily for 3 weeks. There was then a definite decrease in the intensity of the ocular signs and the number of recurrences of keratouveitis. A similar course of treatment was repeated 2 months later.

After the isolation of chlamydia from the eye it was disclosed that the family cat had been suffering from an ocular infection, associated with discharge, about the time of the onset of the patient's symptoms. However, clinical and laboratory tests on this cat for chlamydial or viral infections were all negative.

A clinical examination of the genital tract carried out by Dr E. M. C. Dunlop, Diagnostic Clinic, Moorfields Eye Hospital, showed no abnormality.

On 4 November 1975 the patient was admitted as an emergency to Plaistow Hospital with severe chest pain at rest. For the previous 7 days there had been an increasing dyspnoea on effort. An electrocardiogram showed widespread changes in lead II and lead V2-6. Two hours after admission the patient died of sudden cardiac arrest despite vigorous resuscitation efforts.

\section{NECROPSY}

A coroner's necropsy was carried out by $\mathrm{Dr}$ Alan Grant, of Guy's Hospital, on 5 November 1975. It was found that in the aortic valve area were many layers of fibrin with organisation producing thickening, but weakening of the aortic cusps with herniation of 2 of these. There were also loose deposits of fibrin proximal to the valve ring. Histologically the proximal segments of the coronary arteries showed destruction of the arterial walls. There was thickening of the intima and media of the sinus of Valsalva, with many neutrophils in the media, but mainly lymphocytic infiltration of the intima. There was an atrial destructive arterial lesion which could have been embolic or inflammatory. The aorta showed destruction of the intima and the media. The ostia of the coronary arteries were all narrowed by fibrin deposits. Histological and gross examination of other organs showed no abnormality. Unfortunately, because death occurred unexpectedly and in a hospital where no past records were available, sections of the eyes and internal ears were not taken at the necropsy, and no material was obtained for detection of chlamydia from the various cardiac and other lesions.

LABORATORY INVESTIGATIONS

Repeated haematological and biochemical tests 
showed no abnormality. The levels of blood sugar, urea, cholesterol, calcium, triglycerides, and electrolytes were normal. Liver function tests showed no abnormality. The erythrocyte sedimentation rate was persistently raised to between $30 \mathrm{~mm}$ and $42 \mathrm{~mm}$ in the first hour. $X$-rays of the chest and sinuses were normal, but $x$-ray of the spine showed definite changes in the sacroiliac joints suggestive of ankylosing spondylitis. The Kveim test was negative, as was the Mantoux to $1 / 1000$ in spite of recent BCG vaccination. The rheumatoid arthritis latex test and autoantibody screening were negative. Tests for syphilis including Cardiolipin Wassermann, Venereal disease reference laboratory flocculation test, Reiter's protein complement fixation test, Treponema pallidum haemagglutination test, Treponema pallidum immobilisation test, and Absorbed fluorescent treponemal antibody test were all negative in both the patient and her parents. Examination of cerebrospinal fluid was normal and the CSF Wassermann reaction was negative.

Serum IgM was found to be raised on 3 separate occasions: $245 \mathrm{~mm} / \mathrm{ml}$ on 3 December 1973, 255 $\mathrm{mm} / \mathrm{ml}$ on 15 April 1974 , and $330 \mathrm{~mm} / \mathrm{ml}$ on 19 May 1975. Other serum immunoglobulin concentrations were normal.

Repeated tests from the eyes for virus isolation were all negative. Complement fixation tests showed no increase in the titre of antibody against herpes simplex, varicellazoster, adenovirus, and mumps virus.

The micro-IF test (Wang and Grayston, 1971; Treharne et al., 1972) on the serum obtained on 9 May 1974 showed type-specific antichlamydial antibody at a level of 1/64 against an atypical chlamydia (IOL-207) (Dwyer et al., 1972; Treharne et al., 1972). The level of antichlamydial antibody in this serum against various serotypes of $C$. trachomatis or C. psittaci included in the test (Dwyer et al., 1972; Treharne et al., 1972) was $1 / 8$ or lower. A repeat micro-IF test on 4 February 1975 showed the type-specific antichlamydial antibody at a level of $1 / 32$. Micro-IF tests of the sera obtained from this patient in April, May, and September of 1975 consistently showed a level of 1/64.

In February 1975, after inoculation of a conjunctival specimen collected during an acute exacerbation of keratouveitis into the yolk sac of fertile eggs (Gordon et al., 1969) a chlamydial strain (CPs/GB/ IOL-1959/0) was isolated. Inoculation of the egggrown isolate into irradiated McCoy cells produced intracytoplasmic inclusion bodies similar to those of $C$. psittaci. The isolate produced iodine-negative inclusions in cell culture (Gordon and Quan, 1965) and was resistant to sulphadiazine (Lin and Moulder, 1966). Serotyping by a micro-IF test (Wang et al., 1971) showed that this isolate was not identical with IOL-207, guinea-pig inclusion conjunctivitis agent, feline keratoconjunctivitis agent, iodinenegative lymphogranuloma venereum agent-33-L (Treharne et al., 1972), bovine enzootic abortion of ewes (A22/18), and a strain of C. psittaci isolated from the eye of a patient with follicular conjunctivitis (IOL-395/0). Further characterisation of this isolate is continuing.

Repeated inoculation of irradiated McCoy cell culture (Darougar et al., 1970) and the yolk sac of fertile eggs with specimens collected from the conjunctiva, cornea, urethra, the genital tract, and rectum were all negative.

Micro-IF testing of various samples of sera from this patient using antigens prepared from IOL-207 and her own isolate (IOL-1959) showed a similar titre of antibody (1/64) against both antigens.

With an indirect immunofluorescent staining technique no chalmydial antigens were detected in tissues obtained from the heart, liver, and kidneys that were fixed with formalin for over a year.

\section{Discussion}

The case described above presents several unusual features. The clinical picture of interstitial keratitis and uveitis raised the possibility of several diseases, namely, herpetic or syphilitic infections, Reiter's disease, Vogt-Koyanagi-Harada or Cogan's syndromes, or sarcoidosis. Treatment with topical steroids appeared to have temporary beneficial effect. A good response of the keratouveitis was observed with doxycycline therapy.

Otological symptoms of fluctuating sensorineural hearing loss, tinnitus, and vertigo are suggestive of Menière's syndrome, as distinct from Menière's disease. This is a symptom complex which may be due to a number of disease entities, including Menière's disease itself, the Vogt-Koyanagi-Harada syndrome, Cogan's syndrome, late congenital syphilis, and trauma (perilymph leak (Hinchcliffe, 1973)).

This patient had been treated with indomethacin. The patient's father claimed a few months later that otological symptoms followed her taking an excess dose of indomethacin on one occasion. Sensorineural deafness has occasionally been suggested as a rare side effect of indomethacin treatment (Robinson, 1965; Kinsella et al., 1967). However, other studies have shown that the central nervous system side effects of indomethacin were of a transient nature without sequelae, disappearing completely on cessation of the drug (Rothermich, 1966, 1973). In this patient, although indomethacin was discontinued soon after the onset of the otological symp- 
toms, her clinical condition did not improve over the subsequent months.

The extensive laboratory tests and $x$-rays had ruled out syphilis, sarcoidosis, or collagen vascular disease as the cause of ocular and otological lesions. In the absence of alopecia, vitiligo, and fundus abnormality the diagnosis of Vogt-KoyanagiHarada syndrome could not be sustained.

The chronic fluctuating stromal keratitis and vascularisation of the cornea associated with deafness and cardiovascular abnormalities are highly suggestive of Cogan's syndrome (Eisentein and Taubenhass, 1958; Duke-Elder, 1965; Seel, 1972), though it is difficult to exclude the diagnosis of Reiter's disease because of the associated sacroiliac arthritis. The persistently raised levels of the ESR and IgM in the blood indicated that an infection might be responsible for her various signs. Detection of type-specific antichlamydial antibody by a micro-IF test in her sera and the isolation of a C. psittaci agent from her eyes strongly suggested that chlamydia might be the causative organism.

C. psittaci infection has been shown to be associated with endocarditis. Levison et al. (1971) described 2 cases of endocarditis due to $C$. psittaci causing prominent finger-like vegetations of the heart valve. Ward et al. (1975) discussed 3 patients with C. psittaci endocarditis, 2 of whom died and 1 of whom needed aortic valve replacement. Prominent friable granulations on the heart valve were again a feature of these cases. Regan et al. (personal communication) describe a case of $C$. psittaci endocarditis associated with glomerulonephritis in a patient with a history of prolonged contact with cats and a high level of type-specific IgG and IgM against feline keratoconjunctivitis agent $(C$. psittaci).

In our patient, in the absence of examination of the temporal bones and failure to demonstrate chlamydial antigens in cardiovascular tissues, it is difficult to be as certain about the aetiology of the otological and cardiovascular lesions as about that of the ocular lesions. However, it seems highly probable that these lesions were also caused by the same underlying disease process.

The result of this study suggests that a possible $C$. psittaci infection should always be considered in the diagnosis of any obscure keratouveitis, endocarditis, or Menic̀re's syndrome.

This complicated case was dealt with by many physicians and surgeons in various London hospitals. We thank in particular Dr E. M. C. Dunlop, Mr B. Jay, of the Moorfields Eye Hospital, Mr J. B. Booth of the London Hospital, Dr G. James of the Royal Northern Hospital, and Dr A. Grant of Guy's Hospital for their assistance in preparing this report.

\section{References}

Darougar, S., Kinninson, J. R., and Jones, B. R. (1970). Simplified irradiated McCoy cell-culture for isolation of Chlamydiae. In Trachoma and Related Disorders Caused by Chlamydial Agents, pp. 63-70. Excerpta Medica International Congress Series No. 223.

Dwyer, R. St. C., Treharne, J. D., Jones, B. R., and Herring, J. (1972). Results of micro-immunofluorescence test for the detection of type-specific antibody in certain chlamydial infections. British Journal of Venereal Diseases, 48, 452-459.

Duke-Elder, S. (1965). System of Ophthalmology, Vol. 8, p. 838. Henry Kimpton: London.

Eisentein, B., and Taubenhass, M. (1958). Non-syphilitic interstitial keratitis and bilateral deafness (Cogan's syndrome) associated with cardiovascular disease. New England Journal of Medicine, 258, 1074-1079.

Gelfand, M. L., Kantor, T., and Gorstein, F. (1972). Cogan's syndrome with cardiovascular involvement: aortic insufficiency. Bulletin of the New York Academy of Medicine, 48, 647-660.

Gordon, F. B., Harper, I. A., Quan, A. L., Treharne, J. D., Dwyer, R. St.C., and Garland, J. A. (1969). Detection of Chlamydia (Bedsonia) in certain infections in man. 1. Laboratory procedures: Comparison of yolk sac and cell culture for detection and isolation. Journal of Infectious Diseases, 120, 451-462.

Gordon, F. B., and Quan, A. L. (1965). Occurrence of glycogen in inclusions of the psittacosis-lymphogranuloma venereum-trachoma agents. Journal of Infectious Diseases, 115, 186-196.

Hinchcliffe, R. (1973). In Recent Advances in Otolaryngology, p. 127. Churchill, Livingstone: Edinburgh and London.

Jones, B. R. (1961). Trachoma and allied diseases of the eye. Transactions of the Ophthalmological Society of United Kingdom, 81, 367-378.

Jones, B. R. (1964). Ocular syndromes of TRIC-virus infection and their possible genital significance. British Journal of Venereal Diseases, 40, 3-18.

Kinsella, T. D., Mackenzie, K. R., Kim, S. O., and Johnson, L. G. (1967). Evaluation of indomethacin by a controlled, cross-over technique in 30 patients with ankylosing spondylitis. Canadian Medical Association Journal, 96, 1454-1459.

Lancet, Editorial (1973). Chlamydiae and genital infection. Lancet, 1, 703.

Lancet, Editorial (1977). Chlamydial infections of the eye. Lancet, 2, 857-858.

Levison, D. A., Ward, C., Gurthrie, W., Green, D. M., and Robertson, P. G. C. (1971). Infective endocarditis as part of psittacosis. Lancet, 2, 844-847.

Lin, H. S., and Moulder, J. W. (1966). Patterns of response to sulfadiazine, D-cycloserine and D-alanine in members of the psittacosis group. Journal of Infectious Diseases, 116, 372-376.

Ostler, H. B., Schachter, J., and Dawson, C. R. (1969). Acute follicular conjunctivitis of epizootic origin. Archives of Ophthalmology, 82, 587-591.

Regan, R. J., Dathan, J. R. E., and Treharne, J. D. (1977). Personal communication.

Robinson, R. G. (1965). Indomethacin in rheumatic diseasea clinical assessment. Medical Journal of Australia, 1, 266-269.

Rothermich, N. O. (1966). An extended study of indomethacin: 1. Clinical pharmacology. Journal of the American Medical Association, 195, 531-536.

Rothermich, N. O. (1973). Correspondence. Journal of the American Medical Association, 226, 1471-1472. 
Schachter, J., Arnstein, P., Dawson, C. R., and Hanna, L. (1968). Human follicular conjunctivitis caused by infection with a psittacosis agent. Proceedings of the Society of Experimental Biology and Medicine, 127, 292-295.

Seel, J. (1972). Cogan's syndrome. Interstitial keratitis with sudden deafness. Albrecht v. Graefes Archiv klinische und experimentelle Ophthalmologie, 185, 281-287.

Treharne, J. D., Davey, S. J., Gray, S. J., and Jones, B. R. (1972). Immunological clasification of TRIC agents and of some recently isolated LGV agents by the micro-immuno- fluorescence test. British Journal of Venereal Diseases, 48 18-25.

Wang, S. P., and Grayston, J. T. (1971). Immunological relationships between genital TRIC, LGV and related organisms in a new micro titre-indirect immunofluorescence test. American Journal of Ophthalmolcgy, 70, 367374.

Ward, C., Sagar, H. J., Cooper, D., and Ward, A. M. (1975). Insidious endocarditis caused by Chlamydia psittaci. British Medical Journal, 4, 734-735. 